\title{
Educating on anti-oppressive practice with gender and sexual minority elders: Nursing and social work perspectives
}

\author{
Margaret Pack ${ }^{1}$ and Peter Brown ${ }^{2}$
}

\begin{abstract}
Educational consultant and Registered Social Worker, New Zealand

2 University of Western Sydney, Australia
\end{abstract}

\begin{abstract}
INTRODUCTION: This article relates a common dilemma in professional education out of which developed a collaboration between two health disciplines at a regional Australian university. In a literature review across the two disciplines, the authors drew from social work's teaching knowledge base in an attempt to strengthen the nursing skill base. The intention was to provide students working in the health sector with a consistent theoretical approach and practical tools when working with sexual and gender minorities.

METHOD: As associate professors in social work and nursing, the authors argue on the basis of the teaching and the literature review, for an explicitly anti-oppressive approach to be applied to the education of professionals who work with elders identifying with gender and sexual minorities. Working within an anti-oppressive framework, beginning practitioners in social work and nursing in degree-level education programmes were encouraged to explore their own attitudes including taken-for-granted assumptions often unexplored in the prevailing medical models of care. How different demographics within the lesbian, gay, bisexual, transgender, intersex, queer (LGBTQIA) community experience the health industry is a current issue for educators. There have been increasing challenges expressed by transgender individuals and their concerns over their specific health needs/stigma in rest-home-care facilities, for example.

CONCLUSION: By embedding anti-oppressive principles in our teaching practice, relating to gender and sexual minorities, we acknowledge and open the debate to some of the possibilities/ practicalities/difficulties of advocating for this within a broader multi-disciplinary in small town, rural contexts. The implications for social work and nursing education are discussed.
\end{abstract}

KEYWORDS: gender; diversity; sexual diversity; elders; education; nursing

A questioning of traditional professional responses to the needs of a range of client groups, including elders, those with disabilities, gay and lesbian, and mental health consumers has long been a feature of social work education, more recently fuelled by the rise of the service-user and recovery movements. For nurses, the unacceptability of many aspects of expertknows-best professional practice has created a groundswell for change in which practitioners are re-conceptualising their traditional professional relationships with service users. Teaching nursing and allied health professionals by exploring individual values that impact upon practice with an explicitly anti-oppressive stance is suggested as a way forward. Social workers, due to their training, are well-placed to provide examples of how anti-oppressive practice can apply in the care of elders self-identifying as gender and sexual minorities. Both nursing and social work research needs to include the views of the LGBTQIA community, as 
this could enhance the understanding and application of anti-oppressive practice within a health care system by providing material on the needs and experience of diverse gender and sexual populations within these groups.

LGBTIA is an acronym that stands for a range of sexual and gender diverse identities, including: lesbian, gay, bisexual, transgender, intersex, and queer. The term queer is an umbrella term used to describe an orientation and paradigm that developed out of lesbian and gay experiences, which establishes a theoretical framework for understanding alternative lifestyles and ways of being. In this article we attempt to look at the differing needs of elders who self-identify with the LGBTIA community, mindful that much of the literature has developed from the lesbian and gay experience.

Health systems are often unwelcoming to gender and sexual minorities. Typically, service providers react with anxiety, embarrassment, pity and even go so far as to reject the patient or resident (Irwin, 2007). The literature on elders from gender and sexual minorities which is predominantly focused on gay and lesbian elders, attests to this attitude. Over time, many gay and lesbian elders have been oppressed, both overtly and covertly. As well as direct discrimination, indirect discrimination is associated with health providers' assumptions that patients are heterosexual in their orientation (Hughes, 2007). Clearly, individuals should be offered the same respect as other gender orientated individuals. Aged care facilities may address sexual orientation issues in their policies, yet in practice these policies may not be reflected in the day-to-day quality of the care provided for elder residents who selfidentify with the LGBTIA community.

As authors of this article, both academic heads in two different disciplines, social work and nursing, our teaching at a regional university in the Northern Territory of Australia reflects aspects of the unique environment in which our students and ourselves both live and practise. As a remote and rural location, around $4-5$ hours by air to the nearest main cities of Australia, the present-day Northern Territory is typically seen as originating historically from a frontier society. Archival accounts of living in the Northern Territory describe harsh climatic extremes and landscapes, which include areas of desert, lush tropical jungle and cyclonic weather conditions. Traditionally, living in the Territory was seen as a white man's place, unsuitable for women due to the factors identified above, therefore, an overriding macho ethic has operated locally since white colonial settlement (Bennett, Green, Gilbert, \& Bessarab, 2013). The history of relations with the Aboriginal people is written from a white colonial perspective with little acknowledgement of the Indigenous culture. This invisibility of the Aboriginal people is an issue we have tried to address by introducing readings and textbooks that critique the absence of written accounts of Indigenous history and colonisation in Australia (Bennett et al., 2013). Therefore, in our teaching we endeavour to look with a critical gaze over all approaches to practice where invisibility or marginalisation of any group in society is an issue. Our definition of culture in our teaching is, of necessity, a broad one, encompassing dimensions of age, gender, ethnicity, geographic location, and sexual orientation. Social workers are encouraged in their undergraduate studies to critically reflect on their understanding of colonisation and the monocultural context of their profession. They are also asked to develop an understanding of their own cultural identity including such issues as ethnic background, place of birth, world view and spirituality (Bennett et al., 2013). As many of these dimensions of culture overlap, we endeavour in the classroom to develop students' understanding of the lived experience of culture and illustrate ways in which it impacts on a daily basis on 
our personal lives and professional practice. Educational institutions which offer health care courses of study, we firmly believe, need to include content on these issues and to identify service and support needs of clients who have been systematically marginalised by mainstream White Australia. Most recently the failure to have legislation passed to legalise gay and lesbian marriage through civil union in Australia speaks to an intolerance of difference by overriding dominant heterosexist attitudes and relationships.

\section{Age as aspect of culture}

Where LGBTIA individuals are also ageing, old age makes them at risk of two prevailing sets of negative attitudes: age and sexuality. In the training of healthcare professionals, exploration of individual values that are likely to impact on service delivery, is often sadly lacking in areas such as nursing that has historically have been dominated by medical paradigms. As late as the mid-2000s, Pearson and Vaughan (2005) argued that traditional models based in diagnosis and treatment were still the models on which many nurses base their practice. However, Dahlkemper (2013) points out that, more recently and increasingly, nurses work within relational frameworks of practice.

Social work's focus on emancipatory ideals and social justice concerns, as exemplified in anti-oppressive approaches, offers a way of examining one's own values that inform one's practice as a health professional. Encouraging awareness of one's values is a means of building respect for individual and group differences and provides opportunities for conversations about partnership between those marginalised and the prevailing attitudes of dominance. These conversations between health care providers and LGBTIA elders need to include an acknowledgement and appreciation of the importance of LGBTIA lifestyles and ways of being in intimate relationships. It is critical that healthcare professionals explore the support networks which surround LGBTIA elders collaboratively as part of any assessment and integrated plan of care.

\section{Themes from the research literature on LGBTIA}

In teaching our students about the importance of culture and context to providing high quality service provision in health, we present the evidence-based research literature in our teaching of social work and nursing students, and discuss trends in the literature. Most research studies have focussed on gay men with very few studies focusing on bisexual or transsexual individuals, and the health needs of younger gay and lesbian people. Many studies of gay and lesbian elders have used small samples which may not be representative of sociocultural demographics in the Australian population (Hughes, 2007; Langley, 2001). Therefore, it is difficult to be precise about the numbers of individuals likely to be impacted and the scope of the issue. The social taboos surrounding disclosure and the invisibility of LGBTIA elders make this research even more difficult. It is predictable however, that, with the increase in the number of people moving through into old age in Australia the number of LGBTIA elders will increase and, therefore, their needs require to be planned for both individually and at the broader level of service planning and development. This does not assume that all LGBTIA elders are a homogenous group and share the same needs and preferences. The existence of needs distinct from heterosexual elders have, however, been clearly identified in the research literature and these needs require acknowledgement, discussion and planning for (Irwin, 2007).

Older people who self-identify as LGBTIA are more likely to live alone and are less likely to have children when compared with their heterosexual counterparts. However, this demographic does not necessarily mean that LGBTIA elders will be lonely or isolated 
(Hughes, 2007). There is a need for health and aged care services to better understand and acknowledge older residents' sexual identities to appropriately meet their needs. We must remember too, that not all LGBTIA elders in Australasia are the same and there is great diversity and generational differences within these groups. In particular, those who grew up in an era when their sexual orientation and preference were regarded as against the laws at the time will require great sensitivity on the part of aged care service providers and services in relation to their present needs (Hughes, 2007).

During the submissions to the Productivity Commission Inquiry into Aged Care in Australia, the National Health Alliance proposed that gay and lesbian older adults should be recognised as a special needs group under the Aged Care Act, 1997 (Australian Government, 1997). As a consequence, it was concluded that service providers should receive more appropriate training in catering for the needs of gay and lesbian elders as part of the operationalisation of this Act. How far this specific recommendation is addressed in health care professionals' education, however, remains unclear.

\section{Nursing needs of LGBTIA elders}

Deficiencies in nursing care of LGBTIA elders have been highlighted in an earlier publication by the Royal College of Nursing (2003). Needs identified included concerns about homophobic attitudes of health care workers, fears and consequences about clients being open about their sexual orientation, fears of physical and psychological harm, and concerns about breaches of confidentiality (Royal College of Nursing, 2003). Within the wider LGBTIA community, gay and lesbian elders report experiencing negative and even hostile reactions to their sexual orientation by nurses (Irwin, 2007). Negative reactions might also be more perceived rather than real due to elders' previous experiences of discrimination in other areas of life (Hughes, 2007). There are reports of higher alcohol use related to stress among gay and lesbian elders in the community and they are considered to be more at risk of mental and physical health problems than the general population (Royal College of Nursing, 2003). The gay or lesbian person who is also ageing will be further stigmatised compared to other age groups (Royal College of Nursing, 2003).

For the trans community, it appears not to be simply an attitudinal issue, when health professionals provide services to clients selfidentifying as trans. There is often a lack of provision of appropriate health services, as the needs have not yet been acknowledged in the range of services available, or if they have, there is a poor understanding of the process relating to assessing eligibility for the health services requested, for example, surrounding gender reassignment surgery (Zhang, 2016). In one recent research study, the author interviewed six health professionals using a qualitative approach involving in-depth interviewing in which their work with the transgender and transsexual community in Auckland, New Zealand was inquired about. Contrary to the expectations that professionals are involved at some level in negative stereotyping, the researcher found those interviewed did have a thorough understanding of the differences of working with the transgender/ transgender community and a sensitivity to what was appropriate professional behaviour towards their trans clients (Zhang, 2016). This finding may have been because the method of sampling involved the researcher's networks using a snowballing method of recruitment of participants, which may have led to speaking to those who were already knowledgeable about the effects of negative attitudes to health outcomes (Zhang, 2016).

Part of the problem of identifying the healthcare needs of elders who identify with the wider LGBTIA community relates to definitions of gender, sexuality and family, and relationships within families. Previous research on lesbian, gay and bisexual parents in the New Zealand 
context has recommended that specific protective policies be developed that focus not only on LGB parents, but also their children, regardless of the children's social or sexual identity (Henrickson, n.d.). This recommendation could be extended to the wider population of elders identifying within the LGBTIA community. Healthcare decisions often fail to acknowledge that some LGBTIA elders have children, some conceived while in heterosexual relationships, and that they wish to continue in a close parenting relationship with their children through the life course. LGBTIA in relationships who are co-parents to a same-sex partner's children, or in fostering or whangai relationships, it is argued, must have the opportunity to have those relationships and their parenting roles formally recognised and endorsed by appropriate policies (Henrickson, n.d.). These policies include the decision making of adult children and their LGBTIA parents about residential aged care facilities, and what is appropriate healthcare for them in the later years of life.

\section{Nurses' attitudes towards LGBTIA: Findings from the literature}

The literature in nursing is more focused on the differing needs of gay and lesbian elders within the LGBTIA community. An early study by Rondahl, Innala, and Carlsson (2004) reported that registered nurses expressed more positive attitudes and provided better quality care for lesbian and gay men than assistants in nursing and compared with findings from earlier international studies. However, they suggest that more needs to be done to increase more positive attitudes and therefore enhance their overall wellbeing. Elsewhere, Rondahl's (2009) findings showed that study participants reported very negative attitudes toward gay and lesbian people and some reported feeling anger related to their sexual orientation. Gay and lesbian staff members and partners of patients reported feeling concerned and fearful if they themselves "came out," constantly assessing the risk related to being open about their own sexuality (Rondahl, 2009).

In relation to nurses' and medical students' access to knowledge about the needs of gay and lesbian elders, serious shortcomings have been identified in the research literature (Rondahl, 2009). The aim of the study undertaken by Rondahl (2009) was to look at the students' access to knowledge concerning gay and lesbian elders. Shortcomings in the students' knowledge of the needs of gay and lesbian elders were seen in the student groups surveyed irrespective of education programme, gender or religious belief. Accordingly, the conclusion of the study was that it is likely that heteronormativity will continue to be a feature of all communication, treatment and care if something is not done to address the underlying attitudes of healthcare providers (Rondahl, 2009).

A synthesis of findings from seventeen studies was undertaken by Dorsen (2012) and these highlighted that every study reviewed was found to demonstrate evidence of negative attitudes among health care providers towards gay and lesbian elders. However, Dorsen (2012) noted that there were critical limitations to the studies cited, including that most were quantitative studies, and there were problems and issues with the instruments used to measure attitudes (Dorsen, 2012).

Irwin (2007) recommended that general education of health care professionals through workshops, seminars and attendance at relevant conferences about homosexuality and homophobia should be provided to raise knowledge about their needs as a group (Irwin, 2007). Education about their needs during both undergraduate and postgraduate education is also considered to be important (Irwin, 2007).

Students should be challenged about their attitudes, biases and prejudices, and more senior nursing staff challenged to model 
appropriate attitudes and behaviour. The Attitudes Toward Lesbians and Gay Men (ATLG) Scale (Herek, 1998) is a brief measure of heterosexuals' attitudes toward gay men and lesbians. It is recommended that this scale be used by healthcare professionals as it can be self-administered as a paperbased measure or administered remotely, for example, by telephone. We encourage students to use this type of assessment tool in order to self-assess their own and their peers' attitudes towards gay and lesbian clients.

An anti-oppressive approach to gay and lesbian individuals has been widely used in the teaching of social work (Dominelli, 2002). Arguably, undergraduate and postgraduate nurse education courses should begin to use anti-oppressive theories in the teaching of practice skills to assist in rapport building and engagement with LGBTIA by nurses. Zhang (2016) summarises some of the assessment approaches for working with the trans community that follow anti-oppressive lines by highlighting that having trans-friendly health care providers and facilities is essential as a first step to better health experiences along with written or formalised institutional standards of care (Zhang, 2016, p. 53).

\section{The relevance of anti-oppressive and emancipatory approaches}

Anti-oppressive and emancipatory perspectives focus on addressing institutionalised discrimination in society when one group exerts power over another (Payne, 2005). To understand the power dynamics at play of a predominant group over a group that has become systematically marginalised in society, social workers need to critically reflect on how, structurally and dynamically, this situation arose. Each practitioner, whether social work or nurse, needs to approach these issues by exploring the range of personal, cultural and social factors that can work together to discriminate against individuals and groups in society. Therefore, anti-oppressive theory derives from a social critique and analysis founded in the notion that oppression arises when one group which predominates, exerts their power upon another leading to inequalities in access to resources through the operation of social processes. Dominelli (2002) advocates an analysis of the social identities that produce oppression on a societal level and for this analysis to form the basis of social work practice with marginalised groups in society. Social work is mainly concerned with these disenfranchised groups and the process by which they systematically become marginalised, therefore this critical reflective analysis is essential to social workers and other practitioners learning to practise in an ethical and rights-based way. For example, Price (2008), in her research on gay and lesbian elders with dementia discovered that there is a complex range of factors that dynamically work together on multiple levels and ways to effectively exclude gay and lesbian elders as a population from an holistic model of care. Price (2008, p. 1341) argues that the biomedical model heightens the invisibility of gay and lesbian elders who have a diagnosis of dementia, which means that their social identity becomes more defined by the diagnosis itself rather than aspects of their identity such as spirituality, sexuality and preferred lifestyle:

Furthermore, once a person has dementia. The diagnosis and its presumed personal and public consequences somehow become a person's chief defining characteristics. Other social identities are perceived as less important, or at least less pressing, and are thus extinguished in the observer's eye-a response, perhaps, to the persuasiveness and power of the stereotypes, stigma and discrimination that surround the condition.

The health care environment should be safe for all patients and the services provided, made more widely accessible and user-friendly for gay and lesbian clients. Workplace policies should include expectations around confidentiality of 
information, avoiding comments that presume clients' heterosexuality or use of language that intimidates. There is also a responsibility to challenge other nursing colleagues' negative attitudes where they occur (Royal College of Nursing, 2003). Policies and practices that encourage partners to be included in health care planning meetings and decision making are strongly recommended (Blackwell, 2008; Irwin, 2007). Health care professionals engaged in research will need to focus on LGBTIA health care needs and experiences and make recommendations about how these might need to be met more appropriately (Royal College of Nursing, 2003).

\section{Social work's contribution to the evidence-base on gay and lesbian elders}

Previous research examining the experiences of LGBTIA in Australian health and aged care services from a social work perspective deals mainly with gay and lesbian elders. This research has also revealed direct discrimination and indirect experiences of discrimination (Hughes, 2007). Discrimination in Australia is similarly considered to be both widespread and under-reported (Hughes, 2007). Very similar findings have been reported in the North American context (Stein, Beckerman, \& Sherman, 2010). Research studies undertaken by social workers about gay and lesbian elders in Australia have in the main been small scale, employed a narrative methodology, individual and focus group interviews. From these, direct forms of discrimination have been reported of homophobic and sexist incidents, for example, involving insensitive gynaecological investigations performed without informed consent by a consultant in front of medical students (Hughes, 2007). Indirect experiences of discrimination were described in the attitudes of health care professionals making assumptions of elder clients being heterosexual. Failure to provide gay- and lesbian-friendly services in an Australian study has also been reported (Hughes, 2007) and difficulties accessing appropriate health care have reportedly affected expectations about experiences towards using services in the future (Hughes, 2007). This significant finding, written from a social work perspective, concluded that "attitudes towards identity disclosure in later life were influenced by earlier experiences and perceptions about the risks of disclosure and possible consequences" (Hughes, 2007 p. 205). Variables such as ageism and sexism were equally evident in the narratives of participants suggesting that there were broader social attitudes operating to discriminate on the basis of age and gender, as well as sexual orientation and identity (Hughes, 2007, p. 206).

\section{The role of anti-oppressive practice in social work with elders self-identifying as LGBTIA}

Practice in social work has emphasised a commitment to facilitating client access to equal opportunities. This central tenet of social work practice is confounded, however, by a range of difficulties which include high work/caseloads, hierarchical agency structures and rigid processes that focus on ameliorating individual, rather than group or societal, discrimination (Dominelli, 2002). In focusing on the individual issues as private matters without a broader analysis of societal attitudes, as health social workers we risk reflecting the same marginalisation by the predominant groups back to the clients with whom we practise, and in so doing, inadvertently, and inevitably, maintain the status quo.

Frustration caused by an inability to tackle overriding societal attitudes towards marginalised client groups has led to many social work educators and practitioners leaving the field in search of more serviceuser-focused practice (Dominelli, 2002). In alternative settings, social workers aim to address negative societal attitudes and the power differences that exist in society more 
structurally, to look beyond the individual to address the attitudes that surround client groups marginalised by mainstream attitudes (Langley, 2001). Anti-oppressive practice in social work is designed to address social divisions and structural inequalities in their work across contexts. Social workers know the value of working in collaboration with clients and believe that partnership is an alternative to pathologising top-down approaches. These expert-knows-best models are prevalent in nursing, medicine and psychology. Anti-oppressive approaches challenge the very foundations of what counts as professionalism as the positioning of client and social worker is reformulated on the basis of shared decision-making with service-users having the power to make decisions and choices in their lives (Dominelli, 2002).

Anti-oppressive principles have been applied in social work with gay and lesbian elders (Hughes, 2007; Langley, 2001), however, there are a number of considerations related to LGBTIA that are unique to each group represented within this umbrella term, and questions about the application of antioppressive principles in practice with each group. The first step in developing antioppressive practice is to examine one's own beliefs and acknowledge that heterosexual healthcare workers need to have a greater awareness of their own attitudes and the impact of these values on their practice (Langley, 2001). Once examined, these attitudes can be owned and acknowledged as informing the practitioner's worldview when approaching work with LGBTIA elders. Secondly, there is a call to respond to powerlessness and structural inequality through social workers' activism in influencing agency practice towards social change. Concannon (2009), for example, calls for developing inclusive health and social policies for older LGBTIA by increasing a sense of belonging through citizenship and social inclusion. He advocates for social workers to critique their own power and control as experts and to adopt a model where power is handed over to consumers to promote their self-determination, control over lifestyle and quality of life (Concannon, 2009, p. 407).

The nursing literature emphasises that horizontal violence often occurs amongst nursing colleagues in the workplace where LGBTIA are residents and that this also impacts upon nurses' quality of care (Irwin, 2007). Therefore, addressing the philosophy and role of the organisation is also favoured in an anti-oppressive approach (Irwin, 2007). Critically reflecting upon the internal workplace dynamics, prevailing culture and organisational hierarchies and an antioppressive approach to care, therefore, provide important foci for the education of students in professional programmes of study.

\section{Applications of anti-oppressive practice with elders self-identifying as LGBTIA}

Langley (2001) recommends that social workers and other health practitioners should find ways of validating samesex relationships without requiring that clients disclose their sexuality if it feels uncomfortable for them to do that. Years of "passing" as straight may mean that concealing or downplaying matters relating to sexuality becomes a priority for many gay and lesbian elders and needs to be respected and acknowledged (Langley, 2001). Furthermore, health agency culture may function to unintentionally oppress elders who are gay and lesbian due to heterosexist assumptions that may coalesce around assessment, referral and planning processes. Langley (2001, p. 928), therefore, advocates that health professionals be provided with opportunities to rehearse (during their training) ways of questioning that allow the history of personal relationships to gradually emerge without the need for concealment or denial of one's identity. Open-ended questions such as: "Please describe the relationships that are significant to you" rather than asking about marital status are recommended. Health professionals should 
also be mindful of the use of language (Langley, 2001, p. 928). Nurses need to be encouraged to broaden their theoretical basis for practice both in relation to the range of approaches utilised to guide practice, and in their choice of language. For example, discussing "partners" or "friends" and "community" rather than "husband" or "wife" may be more user-friendly to LGBTIA and make a difference to the experience of the individual patient or resident (Langley, 2001, p. 928). Approaches such as this could be role played in educational settings and workshops before students' entrée into the practice field.

\section{Teaching anti-oppressive practice to facilitate critical reflection}

As educators, we find the place to begin in teaching our students about discrimination is with our own experiences of being discriminated against due to gender (e.g., being a man in a female-dominated nursing profession) and having a feminist orientation to social work practice. We could also discuss how our unquestioning acceptance of heterosexism as the norm, as exemplified in many healthcare agency protocols and assessment and treatment processes, can inadvertently promote the acceptance of subtle (and not so subtle) forms of oppression, and so uphold the status quo. We could go on to discuss how the values of conformity and sameness, embedded in the assessment and treatment process, affects our experiences of working with LGBTIA as practising clinicians. As a nurse-educator (author), I discuss the oppression that can occur between nursing colleagues as a rite of passage from novice to expert nurse. I often relate and discuss being mocked by a female colleague for working in what is traditionally, a predominantly female profession (nursing).

As a social worker, I (author) discuss with social work students the example of advocating for a LGBTIA elder at risk of being made homeless due to being given a lower priority by the local housing authority as a "single" person as no family members lived with her. The wider issue of what constitutes "family" is then critically reflected on and discussed.

The intolerance of gender and sexual diversity is also illustrated as there are rigid protocols in hospitals and aged care facilities around separating men's and women's sleeping quarters. Separation of the genders in aged care facilities' sleeping accommodation itself implies the predominance of ageist and heterosexist values in those contexts. Students are expected to reflect in personal journals how agency protocols and practices serve to uphold rigid gender stereotypes, prevalent in society, that work against the wellbeing of their clients who are LGBTIA. This critical reflective process enables the familiar to become examined as students approach their employing or fieldwork agencies with a more critical gaze.

\section{Implications for the education of health care professionals}

For social workers and nursing professionals, there is a need to provide opportunities for older people to disclose aspects of their sexual identities and to discuss the impact this may have on their preferences for health care delivery. The operation of agency policy assessment protocols will be important for educators to reflect on with health practitioner students as a central part of their training. For example, as a standard part of the assessment, elders may be invited to discuss their gender and sexual identities and relationships using some of the strategies mentioned throughout this article. In a residential care context, LGBTIA elders may require careful and sensitive questioning to provide them with the choice to respond in a way that seems most appropriate and comfortable for them.

As illustrated in previous studies, past experiences of discrimination are likely to 
impact on whether, or how, elders wish to disclose their sexual identity. There are generational differences in relation to what being gay or lesbian means for them in the contexts of their lives that also requires acknowledgement by health care providers. Some older LGBTIA may have had mixed experiences with the LGBTIA communities throughout their lives and this might mean that they prefer to look at alternatives to traditional long-term care and residential facilities. Other variables such as gender, age, ethnicity, disability, or health status, may need fuller exploration, discussion and consideration depending on individual needs. The use of an anti-oppressive stance in health practitioners' interactions with service users may enable older LGBTIA service users to articulate their priorities and the values that have been important to them throughout their lives. Opportunities to disclose identities and relationships that are meaningful require attention during assessment and delivery of, health care services.

\section{Conclusion}

The majority of research studies reviewed concluded that it is essential for health providers to address their own attitudes and also adopt an explicitly anti-oppressive approach in care of LGBTIA elders. These learning opportunities need to be embedded across all professional courses of study. Education providers need to develop LGBTIA friendly environments and curricula so that students can self-critique their own practices in relation to the existing societal heterosexist and heteronormative assumptions they bring to their work with elders who identify as LGBTIA. As part of their practice, students need to consider the overarching values and practices that are espoused by their employing agencies and actively learn methods to report and critique these. Adopting a perspective that transfers power and control towards working with the service-user's narrative of their life and expectations is an underlying assumption of and prerequisite for working with elders who identify as LGBTIA. Due to our experiences in teaching nurses and social workers, we strongly recommended that education of health professionals provides opportunities for practising appropriate assessment and intervention within which LGBTIA elders are able to disclose and express aspects of their identities and relationships, and for these areas to be thoroughly explored. In this goal, the antioppressive and emancipatory approaches that are part of social work theory can guide and inform usefully nursing education practice (Dominelli, 2002; Payne, 2005). Nurses and social work students need to learn that, through the disclosure that they facilitate by such conversations with LGBTIA elders, areas of life previously invisible may become visible in ways that elders themselves can control, discuss and describe. What is appropriate to disclose about their lives, therefore, can be left for LGBTIA elders to decide more proactively (Hughes, 2007; Langley, 2001; Stein et al., 2010).

Although most previous research studies on the health care needs of gay and lesbian elders are based on small samples, and the research on LGBTIA is scarce, the findings presented in a range of qualitative studies highlight the complexity of assessing older LGBTIA needs which are related to their gender and sexual identities (Langley, 2001; Stein et al., 2010; Hughes, 2007). Secondly, and perhaps most importantly to health care providers, awareness of the obstacles to LGBTIA elders' discussion about their lives and social identities, when in contact with health and aged care providers, needs to be addressed. These conversations are often reported as problematic from a service-user viewpoint due to dual societal negative stereotyping about being LGBTIA as well as being older. Therefore, addressing ageism need to be part of the core training of nursing and social work professionals who are involved in elder care. Training opportunities for improved services for LGBTIA elders also need to be incorporated into health care professionals' training in the future. 
Social work's knowledge of anti-oppressive practice has a role to play in modelling alternative practice frameworks for other professional groups such as nursing, to facilitate alternative ways of exploring health professionals' own values about sexual and gender diversity that inevitably impact upon practice. Without an explicitly emancipatory, anti-oppressive approach, healthcare providers and their educators may inadvertently reflect the stereotypes they hope to challenge.

\section{References}

Australian Government. (2007). Aged Care Act, 1997. Retrieved from www.comlaw.gov.au

Bennett, B., Green, S., Gilbert, S., \& Bessarab, D. (2013). Aboriginal and Torres Strait Islander social work. Melbourne, VIC: Palgrave Macmillan.

Blackwell, C.W. (2005). Registered nurses' attitudes toward the protection of gays and lesbians in the workplace: An examination of homophobia and discriminatory beliefs (Unpublished doctoral dissertation). University of Central Florida, Orlando.

Blackwell, C. W. (2008). Registered nurses' attitudes toward the protection of gays and lesbians in the workplace. Journal of Transcultural Nursing, 19(4), 347-353.

Concannon, L. (2009). Developing inclusive health and social care policies for older LGBT citizens. British Journal of Social Work, 39, 403-417.

Dahlkemper, T. R. (Ed.). (2013). Nursing leadership, management, and professional practice for the $L P N / L V N$ in nursing school \& beyond $\left(5^{\text {th }}\right.$ ed.). Philadelphia, PA: F. A. Davis Company.

Dorsen, C. (2012). An integrative review of nurse attitudes towards lesbian, gay, bisexual, and transgender patients. Canadian Journal of Nursing Research, 44(3), 18-43.

Dominelli, L. (2002). Anti-oppressive social work theory and practice. Houndmills, Basingstoke: Palgrave McMillan.

Henrickson, M. (n.d.). Lavender parents. Retrieved from https://www.msd.govt.nz/about-msd-and-our-work/ publications-resources/journals-and-magazines/socialpolicy-journal/spj26/26-lavender-parents-pages68-83. html

Herek, G. M. (1998). Attitudes toward lesbians and gay men Retrieved from http://psychology.ucdavis.edu/rainbow/ html/atlg.html

Hughes, M. (2007). Older lesbians and gays accessing health and aged-care services. Australian Social Work, 60(2), 197-209.

Irwin, L. (2007). Homophobia and heterosexism: Implications for nursing and nursing practice. Advanced Journal of Advanced Nursing, 25(1), 70-76.

Langley, J. (2001). Developing anti-oppressive empowering social work practice with older lesbian women and gay men. British Journal of Social Work, 31, 917-932.

Payne, M. (2005). ( $3^{\text {rd }}$ Ed). Modern social work theory: A critical introduction. Chicago Illinois: Lyceum Books
Pearson, A., \& Vaughan, B. (2005). Nursing models for practice (3rd ed.). Oxford, UK: Butterworth-Heinemann.

Price, E. (2008). Pride or prejudice? Gay men, lesbians and dementia. British Journal of Social Work, 38(7), 1337-1352. Retrieved from http://bjsw.oxfordjournals. org/content/38/7/1337.full

Rondahl, G. (2003). Heteronormativity in a nursing context: Attitudes toward homosexuality and experiences of lesbians and gay men (Unpublished doctoral dissertation). Uppsala University, Sweden.

Rondahl, G., Innala, S., \& Carlsson, M. (2004). Nurses' attitudes towards lesbians and gay men. Journal of Advanced Nursing, 47(4), 386-392.

Rondahl, G. (2009). Students' inadequate knowledge about lesbian, gay, bisexual and transgender persons. International Journal of Nursing Education Scholarship, 6(1), 548-923.

Royal College of Nursing. (2003). The nursing care of lesbian and gay male patients or clients. Guidance for nursing staff. London, UK: Author.

Stein, G. L., Beckerman, N. L., \& Sherman, P. A. (2010). Lesbian and gay elders and long-term care: Identifying the unique and psychosocial perspectives and challenges. Journal of Gerontological Social Work, 53(5), $421-435$.

Zhang, T. (2016). “We are super-duper resilient': Exploring health and professionals' attitudes towards trans people in Auckland (Unpublished master's thesis). Massey University, Albany, Auckland, New Zealand. 